\title{
How to Save the Eloquent Areas in Brain Tumor Surgery State of the Art
}

\section{Prof. Domenico Chirchiglia MD}

\author{
Department of neurosurgery, university of catanzaro, italy \\ chirchiglia@unicz.it
}

\begin{abstract}
In brain tumor surgery the aim is to excise the tumor without creating superior function deficits, so try to spare the so-called eloquent areas. Since the early twentieth century, neurosurgeons like Penfield have paid attention to the preservation of the language areas as well as of the motor pathways during brain surgery. Cortical electrical stimulation and brain mapping were used with good results. The problem was for a long time the invasiveness of the surgery which however foresaw a craniotomy. A few years ago a new and revolutionary technique has established itself. The diffusion tensor Imaging (DTI) is a non-invasive method that includes the use of MRI, allowing the study of white matter and therefore of the cortico-spinal tracts. Tractography is able to distinguish normal nerve fibers from those infiltrated by the tumor, detecting pathological features of white matter such as axonal damage, ischemia, inflammation, edema. The literature is rich in cases related to the use of DTI in brain tumors, the results are excellent if if one thinks of the saving of functionally important brain areas. Therefore, the excision of malignant tumors such as gliomas and glioblastomas is followed by a different and better outcome and a different quality of life. DTI Tractography today represents the best and safest way of preserving the superior cortical functions in brain tumour surgery.
\end{abstract}

Keywords: Brain mapping, awake surgery, tractography, DTI, eloquent areas

\section{INTRODUCTION}

During the years research has tried to be careful not to create or reduce the occurrence of postoperative deficits in brain surgery. The saving of such eloquent areas as language and motility has always been a fundamental and primary objective for the successful outcome of surgery.History teaches us that already in the early years of 1900 the neurosurgeon Penfield created the Montreal procedure according to which the surgical intervention to awake patient and with the electric cortical stimulation and with the help of the brain mapping or to trace the areas to be saved, allowed to make a surgery preserving the eloquent areas $(1,2)$. Penfield used this technique mainly in brain tumor surgery and in drug-resistant epilepsy. Later, George Ojemann continued his cortical electro- stimulation with brain mapping in awake surgery, with excellent results ( 3 ). A study conducted a few years ago on a very large case studies confirms the usefulness of this technique ( 4 ). The limit of cortical stimulation was its invasiveness, hence the need to find a viable and better alternative. A new technique of investigation has been established in recent years, the diffusion tensor, a magnetic resonance instrument through which one can construct three-dimensional images (DTI or Diffusion Tensor Imaging).

\section{DISCUSSION}

The tensor, that is, uses infinitesimal calculus to define geometrical notions of distance, angle and volume and to study the curves of anatomical structures in which a dimension is much greater than the other two (for example the bundles of nerve fibers of the white matter).

The water molecules in a biological tissue are not free to move, because of the presence of the biological membranes and the cellular and infracellulari structures, but precisely for this reason one can observe a 
isotropy of these movements, That is, the water molecules do not move in a particular direction, but they do it in the same measure in all three directions ( 5 ).

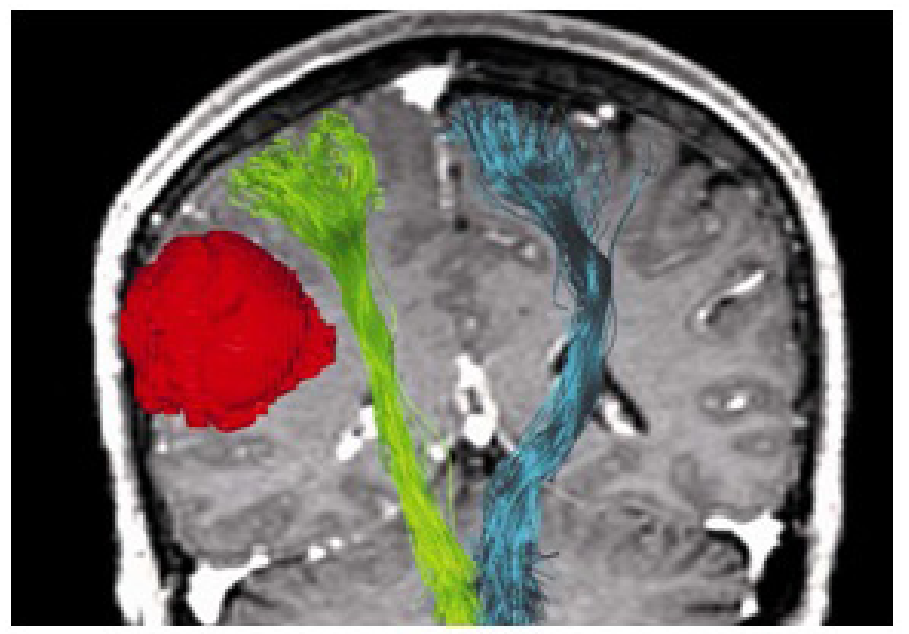

DTI image

Instead, homogeneous anatomic structures, as can be the bundles of nerve fibers, present an anisotropy, that is a peculiar directionality that is reflected in the diffusion of the water molecules and that is used to create the 3d images of the DTI ( 6 ). DTI (Diffusion Tensor Imaging) is an MRI technique that allows the analysis of the diffusive properties and directionality of the flow of water molecules within the tissues in vivo, and is presented as an important tool for the study of the microstructural architecture of cerebral structures in both physiological and pathological conditions. The images are obtained thanks to the use in the RM sequence of intense magnetic field gradient impulses, applied before and after a $180^{\circ}$ radio frequency pulse. A map of the signal strength variation is obtained that provides information about the spread through the direction along which the magnetic field gradient has been applied. DTI data can also be used for the three-dimensional mapping of white matter fibers (tractography) for the in vivo exploration of the anatomical connectivity of the human brain ( 6,7).

The main applications are those in the white matter visualization, to determine the localization, orientation, and anisotropy of the nerve fiber bundles. The architecture of the axon groups in parallel beams and their myelin sheaths facilitate the diffusion of the water molecules preferentially along their main leader. This oriented diffusion is called anisotropic diffusion. The data from the DTI can be used to perform the tractography within the white matter. Algorithms for the detection of fibers can be used to rebuild the path of a fiber along its entire length as the corticospinal tract, which sends the impulses of motor neurons from the motor cortex to the anterior horn motor neurons of the spinal cord.

Tractography is a useful method for measuring deficits in white matter, such as those due to demyelinating disease (adrenoleukodystrophy, SLA, SM) or ageing ( 7 ). Its estimates of the orientation and strength of the fibers are incredibly accurate, and have ample potential implications in the field of neuroscience and cognitive neurobiology.

DTI allows visualization of white matter tracts ( WM tracts) in the immediate vicinity of brain tumors that permit maximum tumor resection while also preserving the eloquent brain areas. The relation of tumors with the white matter tracts (deviation, infiltration, and disruption) has been one the most important initial applications of DTI. The fibers can be infiltrated in normal-appearing areas, and abnormal-appearing areas of the brain can show anatomically intact fibers. In the surgical planning of brain tumors, surgery is aided 
by knowing the proximity and relative position of the tumor to the adjacent WM tracts. Current data shows that tumors that grossly invade areas of functional cortex may still retain functional fiber tracts within the pathological tissue. DTI provides information for tract identification when the white matter tracts are displaced by tumor. Pathological states may affect DTI measurements of intrinsic white matter pathways.

DTI tractography brings complementary information that helps elucidating the complex relationships between the tumor and its surrounding cerebral tissue. Some studies report evidence of intact fiber bundles traversing areas of tumor invasion while others demonstrated displacement of white matter fibers from their normal anatomical position.

The case of a patient is described, affected by glioblastoma located centrally within the left hemisphere, the lesion displaced the corticospinal tracts within the corona radiata medially and anteriorly. So, knowledge of this displacement assisted in preoperative planning by informing the surgeon of the tract's shifted location, thus allowing for adaptation of the surgical corridor to avoid destruction of the communicating white matter bundles. In this instance the tumor was approached from a temporal posterior direction, allowing for aggressive resection of the tumor while avoiding the anteriorly deviated motor fibers ( 7 ). This resulted in postoperative improvement of the patient's hemiparesis, presumably due to the elimination of pressure on the corticospinal tracts. Thanks to the use of the DTI maximal surgical tumor resection has been shown to correlate with longer patient survival and improved long-term functional status representing the main predictor of patient outcome. Diffusion-tensor imaging allowed for the identification of multiple viable white matter pathways within hemispheres involved by tumor. Involvement of white matter tracts represents an important piece of information in planning the surgical approach and in evaluating the extent of a safe resection in patients with brain tumors.

DTI tractography indicates that anatomically intact fibers may be present in abnormal appearing areas of the brain. Careful preoperative planning has to be complemented by intraoperative mapping and monitoring of eloquent cortical areas in order to insure a good neurological outcome of the surgery and valid functional prognostic. In planning this surgery, an equal importance should be attributed to the blood vessels that supply salient areas or tracts with significant functional value. Diffusion tractography of magnetic resonance imaging (MRI), such as diffusion tensor tractography, allows us to visualize white matter tracts in vivo and to study white matter integrity quantitatively. Three dimensional visualization of the white matter fibers such as corticospinal (pyramidal) tract, optic radiation and arcuate fasciculus with relationship to brain tumors such as gliomas is extremely helpful for preoperative evaluation and intraoperative navigation. Using fiber tracking, DTI can develop white matter mapping visualizing components of the pyramidal tract (fibers from foot, hand, face motor areas separately) and making a probabilistic map.

Diffusion tractography is a unique tool to visualize and segment the white matter pathways and one can evaluate the segmented tract quantitatively ( 3,6). Importance of this tool will become more significant in clinical and neuroscience fields in the future. A nod to the limits of DTI: during the diagnostic procedure, which has an average duration of between 30 and 60 minutes, a specific device is used to scan the tissues of the brain and then send the information collected from a computer that processes by returning images to be analyzed. In general, DTI, as the limitations concerning MRI procedures, is contraindicated for specific categories of patients: patients with pacemakers, those who have certain types of prosthetics at the heart valves, and those who have metal implants or foreign metal bodies in some body location. Finally, high-definition fiber tractography (HDFT) is an advanced white matter imaging technique derived from magnetic resonance imaging diffusion data, shown to overcome the limitations of diffusion tensor imaging. Fiber tracking, preferably HDFT, should be part of preoperative planning to achieve maximal extent of resection for longer survival rates in brain tumor patients, while preserving white matter tracts and thus quality of life. 


\section{REFERENCES}

1. Huberfeld $\mathrm{G}$ et al Preoperative and intraoperative neurophysiological investigations for surgical resections in functional areas Neurochirurgie, 2017 Jun;63(3):142-149. doi: 10.1016/j.neuchi.2016.10.008. Epub 2017 May 12.

2. Guenther K Between Clinic and Experiment : Wilder Penfield's Stimulation Reports and the Search for Mind, 1929-55 Can Bull Med Hist,2016 Fall;33(2):281-320. doi: 10.3138/cbmh.33.2.148-27012015. Epub 2016 Sep 7.

3. Ojemann $\mathrm{G}$ et al Cortical language localization in left, dominant hemisphere. An electrical stimulation mapping investigation in 117 patients.1989 J Neurosurg,2008 Feb;108(2):411-21. doi: 10.3171/ JNS/2008/108/2/0411.

4. Signorelli $\mathrm{F}$ et al Indications and limits of intraoperative cortico-subcortical mapping in brain tumor surgery : an analysis of 101 consecutive cases J Neurosurg Sci, 2007 Sep;51(3):113-27

5. Szelenyi A, Bello L et al Intraoperative electrical stimulation in awake craniotomy : methodological aspects of current practice Neurosurg Focus,2010 Feb;28(2):E7. doi: 10.3171/2009.12.FOCUS09237.

6. Hoefnagels FWA et al Impact of gradient number and voxel size on Diffusion Tensor Imaging Tractography for resective brain surgery World Neurosurg,2017 Jun 15. pii: S1878-8750(17)30942-7. doi: 10.1016/j. wneu.2017.06.050. [Epub ahead of print]

7. Aoki S Magnetic resonance diffusion tractography in the brain-its application and limitation Brain Nerve, 2007 May;59(5):467-76.

Citation: Prof. Domenico Chirchiglia, MD. "How to Save the Eloquent Areas in Brain Tumor Surgery: State of the Art". American Research Journal of Neurology, 2(1): 1-4.

Copyright (C) 2018 Prof. Domenico Chirchiglia, MD. This is an open access article distributed under the Creative Commons Attribution License, which permits unrestricted use, distribution, and reproduction in any medium, provided the original work is properly cited. 